\title{
Differential Games Controllers that Confine a System to a Safe Region in the State Space, with Applications to Surge Tank Control
}

\author{
P. Falugi, P.A. Kountouriotis, R.B. Vinter, Fellow, IEEE
}

\begin{abstract}
Surge tanks are units employed in chemical processing to regulate the flow of fluids between reactors. A notable feature of surge tank control is the need to constrain the magnitude of the Maximum Rate of Change (MROC) of the surge tank outflow, since excessive fluctuations in the rate of change of outflow can adversely affect down-stream processing (through disturbance of sediments, initiation of turbulence, etc.). Proportional + Integral controllers, traditionally employed in surge tank control, do not take direct account of the MROC. It is therefore of interest to explore alternative approaches. We show that the surge tank controller design problem naturally fits a differential games framework, proposed by Dupuis and McEneaney, for controlling a system to confine the state to a safe region of the state space. We show furthermore that the differential game arising in this way can be solved by decomposing it into a collection of (one player) optimal control problems. We discuss the implications of this decomposition technique, for the solution of other controller design problems possessing some features of the surge tank controller design problem.
\end{abstract}

Index Terms-Stochastic optimal control; Game Theory; Optimal control; Robust control

\section{INTRODUCTION}

Surge tanks are buffer devices used in chemical processing to prevent excessive flow rate fluctuations, as fluids flow between reactors. The level of the fluid in the surge tank is affected by random variations of the rate of change of inflow and by the control action which governs the rate of change of the outflow. The purpose of the control action is, on the one hand, to restrict the magnitude of the Maximum Rate of Change (MROC) of the surge tank outflow and, on the other, to prolong as far as possible the time before the tank empties or overflows. In surge tank control, MROC is a significant performance indicator. This is because it is the rate of change of outflow, rather than the outflow itself, that adversely affects down-stream processing (through disturbance of sediments, initiation of turbulence, etc.). One can regard states of the surge tank for which the fluid is between the overflow and empty levels as comprising the 'safe region'. While exit from the safe region is tolerated, it is undesirable, because it incurs a cost (for closing down and starting up the unit, or employing an override control). It is therefore appropriate to employ a control strategy that, while not altogether preventing exit, at least renders this an infrequent event.

P. Falugi, P.A. Kountouriotis, R.B. Vinter are with the EEE Department, Imperial College, London, SW7 2AZ, UK. Email: \{p.falugi,pk201,r.vinter\}@imperial.ac.uk.
A simple dynamic model of the surge tank is as follows: denote by $h(t)$ the deviation of the fluid level from its nominal value at time $t$. Then the state of the surge tank at time $t$ is the 2 -vector $x(t)=(h(t), \dot{h}(t))$, comprising the fluid level and the rate of change of fluid level. Assuming uniform cross-section, we obtain a state space equation for the surge tank, relating the state to fluctuations $v(t)$ in the rate of change of inflow and to the rate of change of outflow $u(t)$ :

$$
d x(t) / d t=\left[\begin{array}{ll}
0 & 1 \\
0 & 0
\end{array}\right] x(t)+\left[\begin{array}{l}
0 \\
1
\end{array}\right](-u(t)+v(t)) .
$$

The constraints that the surge tank must neither overflow or empty are expressible (in normalized units) as

$$
-1<x_{1}(t)<+1
$$

Permitted tolerances on the MROC index are captured by the additional constraint on the outflow:

$$
-1 \leq u(t) \leq+1 .
$$

Here $u(t)$ is interpreted as the control signal and $v(t)$ as a disturbance signal.

Traditionally, proportional + integral controllers have been used for surge tank control which aim to regulate the fluid level about a set point [3], [8]. Reducing fluctuations in the rate of change of outflow can, to some extent, be achieved by detuning the controllers. Model predictive schemes have also been proposed [5]. But other kinds of controllers have been developed, to take more direct account of the MROC constraint. McDonald [20] and subsequently Kantor [18] proposed non-linear controllers based on the calculation of a controller that minimizes the maximum rate of change of outflow (the MROC index) for a special class of disturbances (a single step change in the rate of change of inflow). This controller alone allows the surge tank to completely fill or empty over the chosen time horizon; for this reason it is modified by the addition of a sliding mode control term to reduce the frequency or such events. We might seek to improve on this approach for the following reasons. First, it would appear to be more natural to incorporate MROC as a constraint. (The maximum rate of change of outflow should be kept below a specified threshold to avoid disruption to downstream processing.) The McDonald/Kantor approach, based on minimizing MROC, places undue emphasis on reducing MROC, even below the required threshold, instead of on preventing overflow or emptying of the surge tank. Second, 
the controller is designed to take account of just one kind of disturbance.

In [12], Dupuis and McEneaney give careful consideration to the appropriate choice of cost, for problems of controlling a small-noise stochastic control system to influence the exit time of the state from a safe region.We refer to such problems as 'exit time' problems. It is observed that maximizing the expected exit time is not necessarily the most sensible strategy, because the resulting probability of an early exit might be unacceptably high. Instead, these authors propose a cost, parameterized by a risk averseness parameter $\theta$, that takes account of the degree to which an early exit can be tolerated. Conditions are given under which the value function of the stochastic control problem can be characterized as a viscosity solution to the Hamilton Jacobi equation for a given value of $\theta$ and (when the risk averse cost is appropriately scaled by the noise intensity parameter $\epsilon$ ) under which the log transformed value function converges to that of a differential game as $\epsilon$ vanishes.

The aim of this paper is to solve the surge tank controller design problem within the Dupuis/McEneaney framework. We also discuss how the proposed methodology might be employed to generate controllers for related systems. The solution has been announced in [10]. The present paper gives full details of the underlying analysis. This framework appears to offer a number of advantages over earlier proposed approach es that explicitly account of the MROC measure of performance: it yields a controller that maximizes a cost function related to the exit time from the safe region, subject to the constraint that MROC must remain within specified limits. It also implicitly takes account of a more diverse set of disturbance signals than the single step disturbances considered by McDonald and Kantor.

The exit problems of interest here can be equivalently described as stochastic control problems with vanishingly small noise intensity or as differential games problems. (The value functions of the former and the upper values of the latter are the same.) This equivalence is established in [12], and generalized in [13] to cover systems where the noise enters only some of the state components, of which the surge tank system is an example. See also [17] for an expository account of some of the underlying ideas. We may therefore seek to solve either kind of problem, in relation to surge tank control. In this paper we focus on the differential game. This is because we can exploit a special feature of this particular differential game, which greatly facilitates its solution: the problem decomposes into a finite collection of optimal control problems. To be more precise, the upper value of the differential game is the lower envelope of the value functions of these optimal control problems.

Besides providing a solution to the surge tank control design problem interpreted as a differential game, we shall discuss the broader implications of the solution technique employed. One special feature of the surge tank system permitting the decomposition of the differential game into a collection of optimal control problems is its monotonicity properties in relation to the different modes of exit from the safe region. For example, given any fixed disturbance history and initial state the effect of increasing the outflow is to reduce the fluid level (and therefore of increasing the time when overflow occurs). Not only for the surge tank controller design problem, but for other exit problems for which the underlying control systems possess similar monotonicity properties, control design strategies can be devised which, like model predictive control schemes [19], involve on-line optimization. But now they involve the solution of not one, but several, optimal control problems. We give conditions for such a scheme to yield the solution to the differential game. Even in some situations when these conditions cannot be directly verified, this optimization based control can be implemented as a sub-optimal scheme, and bounds can be developed governing its cost. We illustrate this point by considering a higher dimensional variant on the surge tank controller design problem.

There is a substantial literature on the numerical solution of the Hamilton Jacobi equations arising in optimal control and differential games [21], [4]. Attention has been given, in particular, to computation saving schemes for control systems with higher dimensional state spaces [7], [11], [1], [15], [22], of which variants of the surge tank controller design problem involving colored noise disturbances are examples. We point out that the explicit solution provided in this paper to an exit problem arising in process systems, besides providing a new paradigm for surge tank control, has potential use as a benchmark for the application of generic Hamilton Jacobi equation solvers. Furthermore, the surge tank problem focuses on the prevalence in applications of stochastic systems where the noise signal affects directly only certain of the state variables and the classical boundary conditions on the relevant solutions to the Hamilton Jacobi equations are violated at boundary points that are not exit points, and the need for generic Hamilton Jacobi equation solvers that take account of such degeneracies.

\section{A Differential Games Formulation of the State CONFINEMENT PROBLEM}

In this section we describe a differential games formulation, employed by [12], of the problem of controlling a dynamic system with disturbance input to maximize some measure of the exit time of the state from a subset of the state space, interpreted as a safe region. We show presently how this formulation can be employed in surge tank control. The dynamic system is modelled as

$$
\left\{\begin{array}{l}
d x(t) / d t=f(x(t), u(t))+\sigma(x(t)) v(t) \\
x(0)=x_{0}
\end{array}\right.
$$

relating the state trajectory $x($.$) to the control functions u($. and $v($.$) applied by the two players. The data comprise$ functions $f: R^{n} \times R^{m_{1}} \rightarrow R^{n}, \sigma: R^{n} \rightarrow R^{n \times m_{2}}$, sets $A \subset R^{n}$ and $\Omega \subset R^{m_{1}}$ and a vector $x_{0} \in A$. It is assumed that

(H1): There exists $K>0$ such that

$$
\left|f(x, u)-f\left(x^{\prime}, u\right)\right|+\left|\sigma(x)-\sigma\left(x^{\prime}\right)\right| \leq K\left|x-x^{\prime}\right|
$$

for all $x, x^{\prime} \in R^{n}$ and $u \in \Omega$. 
(H2): $\Omega$ is a compact set and $A$ is an open set with representation:

$$
A=\left\{x \in R^{n} \mid h^{1}(x)<0, \ldots, h^{r}(x)<0\right\}
$$

in which, for each $j, h^{j}():. R^{n} \rightarrow R$ is a given $C^{1}$ function satisfying the condition $\left|\nabla h^{j}(x)\right| \neq 0$ at points $x$ where $h^{j}(x)=0$.

Denote by $\mathcal{U}$ and $\mathcal{V}$ the spaces of open loop controls for the $u$ and the $v$ players respectively:

$$
\begin{aligned}
\mathcal{U}:= & \left\{\text { measurable functions } u:[0, \infty) \rightarrow R^{m_{1}}\right. \\
& \text { s.t. } u(t) \in \Omega \text { a.e. }\} \\
\mathcal{V}:= & L^{2}\left([0, \infty) ; R^{m_{2}}\right) .
\end{aligned}
$$

Following Elliott and Kalton [14], we define the space $\Phi$ of closed loop controls for the $u$ player to be

$$
\Phi:=\{\text { non-anticipative mappings } \phi(.): \mathcal{V} \rightarrow \mathcal{U}\} .
$$

Here, a 'non-anticipative mapping' $\phi():. \mathcal{V} \rightarrow \mathcal{U}$ means a mapping such that, for each pair of open loop controls $v, v^{\prime} \in$ $\mathcal{V}$ and each $T \geq 0$, we have:

' $\left.v\right|_{[0, T]}=\left.v^{\prime}\right|_{[0, T]}$ a.e.' implies ' $\left.\phi(v)\right|_{[0, T]}=\left.\phi\left(v^{\prime}\right)\right|_{[0, T]}$ a.e.' .

The differential game is

$$
\left(P_{x_{0}}\right)\left\{\begin{array}{l}
\text { Maximize } \inf _{v \in \mathcal{V}} J\left(\phi(v(.)), v(.) ; x_{0}\right) \\
\text { over } \phi(.) \in \Phi
\end{array}\right.
$$

where the payoff function $J\left(., ; x_{0}\right): \mathcal{U} \times \mathcal{V} \rightarrow R \cup\{+\infty\}$ is

$$
J\left(u(.), v(.) ; x_{0}\right)=\int_{0}^{\tau(x(.))}\left(\frac{1}{2}|v(t)|^{2}+\theta\right) d t .
$$

Here, $\theta \geq 0$ is a design parameter and $\tau(x()$.$) denotes the$ first exit time from $A$ of the solution $x($.$) to (1).$

The payoff $J\left(u(),. v(.) ; x_{0}\right)$ is a weighted sum of the energy expended by the $v$ player to force an exit from the region $A$ (when the $u$ player employs the closed loop control $\phi($.$) ), and$ of the exit time.

The reason for formulating the differential game in terms of non-anticipative controls for the $u$ player, instead of in terms of a control defined by a state feedback relation

$$
u \in \chi(x),
$$

is to circumvent the difficulty that, for non-Lipschitz functions $\chi($.$) , the solution to \dot{x}(t)=f(x(t), \chi(x(t)))+\sigma(x(t)) v(t)$, for given $v(.) \in \mathcal{V}$ and initial state $x(0)=x_{0}$, is possibly nonunique or may fail to exist, thereby leaving ambiguous the meaning of 'state trajectory corresponding to a given $v(.) \in \mathcal{V}$ and initial state $x_{0}$ '.

Often, however, discontinuous state feedback relations can be interpreted as non-anticipative controls for the $u$ player according to the following definition.

Definition 2.1: Take a set-valued function $\chi():. R^{n} \rightsquigarrow$ $R^{m_{1}}$ and an initial state $x_{0} \in A$. A closed loop control $\phi(.) \in$ $\Phi$ is said to be compatible with $\chi($.$) if, for every v(.) \in \mathcal{V}$, the state trajectory $x($.$) corresponding to u()=.\phi(v()),. v($. and $x_{0}$ satisfies

$$
u(t) \in \chi(x(t)) \quad \text { for a.e. } t \in[0, \infty) .
$$

General conditions ([9],[6]) are known for the existence of closed loop controls compatible with a given state feedback.

Proposition 2.2: Assume (H1) and (H2). Take any set valued function $\chi: R^{n} \rightsquigarrow \Omega$ and initial state $x_{0} \in R^{n}$. Assume that

- the set $\operatorname{Graph}\{\chi()$.$\} is closed, where$

$$
\operatorname{Graph}\{\chi(.)\}:=\left\{(u, x) \in \Omega \times R^{n} \mid u \in \chi(x)\right\} .
$$

Then there exists a closed loop control $\phi(.) \in \Phi$ which is compatible with $\chi($.$) .$

Define the upper value $W():. A \rightarrow R \cup\{+\infty\}$ for the family of differential games $\left\{\left(P_{x}\right) \mid x \in A\right\}$ to be the function

$$
W(x)=\sup _{\phi(.) \in \Phi} \inf _{v \in \mathcal{V}} J(\phi(v(.)), v(.) ; x) \quad \text { for } x \in A .
$$

We set $W(x)=+\infty$ if there exists $\phi(.) \in \Phi$ for which no open-loop $v$-control can be found forcing an exit from $A$.

Dupuis and McEneaney have investigated the connection between the differential game and the following stochastic control problem:

$$
\left(P_{x_{0}}^{\epsilon}\right)\left\{\begin{array}{l}
\text { Minimize } E_{x_{0}} \exp \{-\theta \tau(x(.)) / \epsilon\} \\
\text { over measurable control processes } u(.) \text { such that } \\
d x(t)=f(x(t), u(t)) d t+\epsilon^{\frac{1}{2}} \sigma(x(t)) d B(t) \\
x(0)=x_{0} \\
u(t) \in \Omega
\end{array}\right.
$$

in the limit as the noise intensity parameter $\epsilon$ vanishes [12]. See also [13].

Now the evolution of the state is governed by a stochastic differential equation, driven by an $R^{m_{2}}$-valued Brownian motion $B$ (integrated 'continuous time white noise'). 'Measurable' control processes are taken to be $\Omega$ valued processes that are progressively measurable with respect to a filtration induced by the Brownian motion, on some appropriate measure space. $\tau(x()$.$) denotes the first exit time from A$ of the solution $x($.$) to the stochastic differential equation with initial$ state $x_{0}$ and for the given (non-anticipative) control process $u($.$) . A control process is sought to minimize$

$$
E_{x_{0}} \exp \{-\theta \tau(x(.)) / \epsilon\} .
$$

The subscript on the expectation operator emphasizes the specified initial state. The constant $\theta$ is that appearing in the payoff function (3). The positive number $\epsilon$ is the noise intensity parameter appearing in the stochastic differential equation above. It is introduced into the cost in such a manner as to permit an asymptotic analysis of minimizing controls, in the limit as $\epsilon \downarrow 0$.

Cost functions of this kind are referred to as 'risk averse' costs for exit time, and can be thought of as refinements of the expected exit time criterion. Risk averse costs have received considerable attention in the literature [24], [12], [13]. Suffice it to mention here that, for small $\theta$, the effect of minimizing the cost approximates that of maximizing the expected exit time. Increasing $\theta$ produces a control for which the probability of an early exit is lessened, at the price of a reduction in the average exit time. 
Fix $\epsilon>0$. The value function $\tilde{V}^{\epsilon}: A \rightarrow R \cup\{+\infty\}$ for the family of problems $\left\{\left(P_{x}^{\epsilon}\right) \mid x \in A\right\}$ is the function

$$
\tilde{V}^{\epsilon}(x)=\inf E_{x} \exp \{-\theta \tau(x(.)) / \epsilon\},
$$

for $x \in A$. The infimum is taken over measurable control processes $u($.$) (interpreted as above). \tau(x()$.$) is the first exit$ time of the solution $x($.$) to the stochastic differential equation$ for initial state $x$ and the given $u($.$) . We set \tilde{V}^{\epsilon}(x)=0$ if, for all measurable controls, state trajectories $x($.$) (with initial$ state $x$ ) remain in $A$ for all time, with probability one.

The link between the differential game and the stochastic optimal control problem is as follows [12]: under an additional non-degeneracy hypothesis

$$
\operatorname{det}\left\{\sigma(x) \sigma^{T}(x)\right\} \neq 0 \text { for all } x \in R^{n},
$$

on the control system and a regularity hypothesis on the boundary of $A$, it is known that the value function $W($.$) of$ the differential game is the unique viscosity solution (in the space of continuous functions vanishing on the boundary of $A$ ) of the Isaacs equation

$$
\begin{aligned}
\max _{u \in \Omega} & \min _{v \in R^{m_{2}}}\left\{\nabla_{x} W(x)(f(x, u)\right. \\
& \left.+\sigma(x) v)+(1 / 2)|v|^{2}\right\}+\theta=0 .
\end{aligned}
$$

Now write $\tilde{W}^{\epsilon}($.$) for the scaled, \log$ transformed value function of the stochastic optimal control problem:

$$
\tilde{W}^{\epsilon}(x):=-\epsilon \ln \tilde{V}^{\epsilon}(x) .
$$

Then $\tilde{W}^{\epsilon}($.$) converges pointwise, as \epsilon \downarrow 0$, to the viscosity solution of the Isaacs equation in the specified function space, which therefore coincides with $W($.$) .$

Since the logarithmic transformation is monotone, maximizing $-\epsilon \ln \left\{E_{x_{0}} \exp (-\theta \tau(x().) / \epsilon)\right\}$ produces the same optimal controls as minimizing $E_{x_{0}} \exp (-\theta \tau(x().) / \epsilon)$. The analysis referred to above suggests then that solving the differential game is an approach to solving the stochastic control problem for small $\epsilon$. The non-degeneracy hypothesis (5) is not satisfied by the surge tank control system, because the dimension of the disturbance variable $v$ is smaller than that of the state variable $x$. In such cases, the relationship of the two problems with the Hamilton Jacobi equation is not a straightforward one but, nonetheless, it is known [13] that the log transformed value function of the stochastic control problem still coincides with the upper value of the game, in the limit as $\epsilon$ vanishes. In any event, the differential game formulation of the control design problem ('maximize the minimum disturbance energy required to force a violation of the state constraint') has a validity independent of any such relationship.

\section{Associated Optimal Control Problems}

For certain differential games, of which the surge tank design problem formulated as a differential game is an example, the solution decomposes into the solution of a collection of $r$ optimal control problems, which we now describe.

The $j$ 'th optimal control problem is obtained from the differential game by modifying the differential game in two ways. First, the state constraint set $A$, which we recall involves $r$ state constraint functionals, is replaced by the larger set $A^{j}$ which results when we discard all but the $j$ 'th state constraint functional. Thus

$$
A^{j}=\left\{x \in R^{n} \mid h^{j}(x)<0\right\} .
$$

Second, the differential game is reduced to a (one player) optimal control problem by freezing the control action of the $u$ control player at some $u_{j} \in \Omega$. The selected control value $u_{j}$ has the interpretation that it drives the state away from the boundary of $A^{j}$ at the maximum rate. (In the case of the surge tank problem, $A^{1}$ is the state constraint set expressing the requirement 'the surge tank level must not overflow' and the corresponding frozen control value $u_{1}$ gives rise to the maximum rate of outflow, while $A^{2}$ is the state constraint set expressing 'the surge tank level must not empty' and $u_{2}$ gives rise to the minimum rate of outflow.)

For arbitrary initial state $x_{0} \in A^{j}$, the $j$ 'th optimal control problem is taken to be:

$$
\left(P_{x_{0}}^{j}\right)\left\{\begin{array}{l}
\text { Minimize } \int_{0}^{\tau}\left(\frac{1}{2}|v(t)|^{2}+\theta\right) d t \\
\text { over } \tau \geq 0 \text { and } v \in L^{2}\left([0, \tau] ; R^{m_{2}}\right) \text { satisfying } \\
\dot{x}(t)=f\left(x(t), u_{j}\right)+\sigma(x(t)) v(t) \quad \text { a.e. on }[0, \infty) \\
x(0)=x_{0}, h^{j}(x(\tau))=0 .
\end{array}\right.
$$

Notice that $\left(P_{x_{0}}^{j}\right)$ has been posed as a standard free final-time optimal control problem, for which the 'decision variable' is a couple $\left(\tau, v():.[0, \tau] \rightarrow R^{m_{2}}\right)$ comprising the terminal time $\tau$ and a square-integrable function $v($.$) on [0, \tau]$. The state trajectory $x():.[0, \tau] \rightarrow R^{n}$ associated with a given $(\tau, v()$.$) ,$ and with initial value $x_{0}$, is required to satisfy the $j$ 'th endpoint constraint $h^{j}(x(\tau))=0$. If $\left(P_{x_{0}}^{j}\right)$ has a minimizer $(\bar{\tau}, \bar{v}()$. with state trajectory $\bar{x}($.$) , then \bar{\tau}$ will always be the first exit time of $\bar{x}($.$) from A^{j}$ in the case $\theta>0$, and can be replaced by the first exit time in the case $\theta=0$.

The value function $V^{j}():. A^{j} \rightarrow R \cup\{+\infty\}$ evaluated at $x_{0}$, now regarded as a generic point in $A^{j}$, is defined to be the infimum cost for $P_{x_{0}}^{j}$.

For the differential game considered here, each $V^{j}($.$) will$ be finite valued. This implies that, for each $x \in A^{j}$ there exist a pair $(\tau, v()$.$) satisfying the constraints of \left(P_{x}^{j}\right)$. We can then show, by means of a standard weak sequential compactness analysis making use of hypotheses $(H 1)$ and $(H 2)$, and of the facts that the dynamics are linear in $v$ and the cost integrand is coercive, that $\left(P_{x}^{j}\right)$ has a minimizer. (c.f.([23], Section 2.8).)

\section{Decomposition Conditions}

In this section we give conditions under which the solution of the differential game decomposes into the problem of computing the value functions of a finite collection of (one player) optimal control problems. These conditions axiomatize the special properties of the surge tank controller design problem formulated as a differential game, which facilitate its solution.

Recall that optimal control problem $\left(P_{x}^{j}\right)$ is associated with the state constraint set $A^{j}$, and involves a 'frozen' $u$ player control value $u_{j}$ which drives the state away from the boundary of $A^{j}$ at a maximum rate. The first condition, which is further discussed in Section 7 below, makes precise this property:

(C1): Take any $x_{0} \in R^{n}$. Take also any open loop controls $u(.) \in \mathcal{U}$ and $v(.) \in \mathcal{V}$. Let $x($.$) be the state trajectory$ 
with initial state $x_{0}$, corresponding to controls $u($.$) and$ $v($.$) , and let x^{\prime}($.$) be the state trajectory with initial state$ $x_{0}$, corresponding to controls $u^{\prime}(.) \equiv u_{j}$ and $v($.$) . Then$

$$
h^{j}\left(x^{\prime}(t)\right) \leq h^{j}(x(t)) \quad \text { for all } t \geq 0 .
$$

The remaining conditions concern the optimal control problems and properties of their value functions $V^{j}(),. j=$ $1, \ldots, r$. One such condition is a boundary condition for $V^{j}($.$) .$ This asserts that $V^{j}($.$) is required to vanish merely on the j$ 'th 'exit boundary'

$$
\begin{aligned}
\partial A_{\text {exit }}^{j}= & \left\{x(\tau) \mid x(.):[0, \tau] \rightarrow R^{n}\right. \text { is a trajectory } \\
& \text { such that } \left.x(t) \in A^{j} \text { for } t \in[0, \tau) \text { and } x(\tau) \notin A^{j}\right\},
\end{aligned}
$$

and not on the whole boundary $\left\{x \in R^{n} \mid h^{j}(x)=0\right\}$ of $A^{j}$.

The need for a boundary condition of this nature is clear from consideration of the surge tank problem. The $j=1$ state constraint set is

$$
\left\{x=\left(x_{1}, x_{2}\right) \in R^{2} \mid x_{1}<1\right\}
$$

with boundary $\left\{x=\left(x_{1}, x_{2}\right) \in R^{2} \mid x_{1}=1,-\infty<\right.$ $\left.x_{2}<+\infty\right\}$. However $V^{1}($.) cannot vanish on the subset $\left\{x=\left(x_{1}, x_{2}\right) \in R^{2} \mid x_{1}=1,-\infty<x_{2}<0\right\}$. More precisely, $V^{1}($.) cannot have a continuous extension to the closure of $A^{1}$ that vanishes on this subset, because the surge tank can never first overflow at a time when the rate at which the level is changing is strictly negative. The exit boundary is $\left\{x=\left(x_{1}, x_{2}\right) \in R^{2} \mid x_{1}=1, x_{2} \geq 0\right\}$; that is, the complement of $\left\{x=\left(x_{1}, x_{2}\right) \in R^{2} \mid x_{1}=1,-\infty<x_{2}<0\right\}$ in the boundary of $A^{1}$.

The next set of conditions are that the $V^{j}($.$) 's possess$ certain regularity properties, and that they are solutions of the Hamilton Jacobi equation which vanish on the exit boundary. For each $j$, the conditions are

(C2): $V^{j}($.$) is continuously differentiable on \left\{x \in A^{j} \mid V^{j}(x)>\right.$ $0\}$.

(C3): $V^{j}($.$) has a continuous extension to the closure of A^{j}$, which vanishes on $\partial A_{\text {exit }}^{j}$.

(C4): $\min \left\{\nabla_{x} V^{j}(x)\left[f\left(x, u_{j}\right)+\sigma(x) v\right]+\frac{1}{2}|v|^{2}+\theta \mid v \in\right.$ $\left.R^{m_{2}}\right\}=0$

$x \in\left\{x^{\prime} \in A \mid V^{j}\left(x^{\prime}\right)>0\right\}$.

The final two conditions concern the relations of the optimal control problems to one another. They are expressed in terms of the notation: for $x \in A$

$$
I(x):=\left\{j \mid V^{j}(x)=\min _{j^{\prime}} V^{j^{\prime}}(x)\right\} .
$$

(C5): Take any $x_{0} \in A$ and $j \in I\left(x_{0}\right)$. If $\bar{x}():.[0, \tau] \rightarrow R^{n}$ is an optimal state trajectory for $\left(P^{j}\left(x_{0}\right)\right)$, then $\bar{x}(t) \in$ $A$ for all $t \in(0, \bar{\tau})$. In addition, if $V^{j}\left(x_{0}\right)=0$ then $V^{j^{\prime}}(x)>0$ for all $j^{\prime} \neq j$.

(Notice that state trajectories for $\left(P_{x_{0}}^{j}\right)$ evolve in $A^{j}$. The above condition requires that, if $j \in I\left(x_{0}\right)$ then they are confined to the subset $A \subset A^{j}$.)

(C6): Take any point $x \in A$ and $j, j^{\prime} \in I(x)$ such that $j \neq j^{\prime}$. Then, for any $u \in \overline{\mathrm{co}}\left\{u_{k} \mid k \in I(x)\right\}$ (where co denotes the closed convex hull) and $v \in R^{m_{2}}$ such that

$\nabla_{x} V^{j}(x)(f(x, u)+\sigma(x) v)=\nabla_{x} V^{j^{\prime}}(x)(f(x, u)+\sigma(x) v)$, we have $\nabla_{x} V^{j}(x)(f(x, u)+\sigma(x) v) \geq-\frac{1}{2}|v|^{2}-\theta$.

The solution to the differential game will be expressible as a discontinuous state feedback relation, with a switching surface comprising states at which two or more of the associated optimal control problems have the same minimum costs. The value function for the differential game will be the lower envelope of the value functions for the optimal control problems. This last condition restricts the rate of decrease of the value function for the differential game along trajectories that dwell in the switching surface.

\section{Solution of the Differential Game under the DECOMPOSIBILITY CONDITIONS}

In this section we give a description of the solution to the differential game under the conditions listed in the previous section. The following theorem asserts, in precise terms, the optimality of any closed loop strategy compatible with a state feedback control with the property: the feedback relation takes the value $u_{j}$ at points $x$ in the state space such that $V^{j}(x)<V^{j^{\prime}}(x)$ for all $j^{\prime} \neq j$. These conditions, while restrictive, are satisfied in the case of the surge tank controller design problem; their role here is to highlight the distinctive features of this problem that justify this decomposition.

Theorem 5.1: Consider the differential game $\left(P_{x_{0}}\right)$ for some given initial state $x_{0} \in A$. Assume that conditions $(H 1)$ - $(H 2)$ and $(C 1)-(C 6)$ are satisfied. For $j=1, \ldots, r$ let $u_{j}$ be the fixed control value associated with the optimal control problem $\left(P_{x_{0}}^{j}\right)$, and let $V^{j}($.$) be the value function. Define$ $V():. A \rightarrow R$ to be the function

$$
V(x):=\min \left\{V^{1}(x), \ldots, V^{r}(x)\right\} \quad \text { for } x \in A .
$$

Then

$$
\max _{\phi \in \Phi} \inf _{v \in \mathcal{V}} J(\phi(v(.)), v(.) ; x)=V(x) \quad \text { for all } x \in A
$$

(i.e., $\mathrm{V}($.$) is the value function for the family of differential$ games $\left.\left\{\left(P_{x}\right) \mid x \in A\right\}\right)$. Furthermore, for any initial state $x_{0} \in A$, there exists a closed loop control $\bar{\phi}(.) \in \Phi$ (it will depend on $x_{0}$ ), compatible with the state feedback relation $\bar{\chi}():. R^{n} \rightarrow \Omega$ :

$$
\bar{\chi}(x)=\overline{\mathrm{co}}\left\{u_{j} \mid V^{j}(x)=V(x)\right\} \quad \text { for } x \in A,
$$

and any such closed loop control is a maximizer for $\left(P_{x_{0}}\right)$. A proof of Thm. 5.1 appears in the Appendix. It might seem surprising that the theorem statement makes reference to the initial state $x_{0}$, since the state feedback $\chi($.$) , in terms of which$ the solution is expressed, is independent of $x_{0}$. The need to consider the initial state arises for technical reasons: strictly speaking it is not the feedback relation that solves the game problem but some closed loop control $\phi($.$) compatible with$ this feedback relation. It may happen that the way the closed loop control selects controls consistent with the set valued feedback relation depends on the initial condition. 


\section{Vi. Application to the Surge Tank Controller DESIGN PROBLEM}

The surge tank controller design problem described in the introduction conforms to the differential game $\left(P_{x_{0}}\right)$ of Section 2, when we take $r=2, n=2, m_{1}=m_{2}=1$, $\Omega=[-1,+1]$,

$$
f(x, u)=F x(t)-g u(t) \text { and } \sigma(x)=g,
$$

where

$$
F=\left[\begin{array}{ll}
0 & 1 \\
0 & 0
\end{array}\right] \text { and } g=\left[\begin{array}{l}
0 \\
1
\end{array}\right]
$$

The state constraint set is $A=\left\{\left(x_{1}, x_{2}\right) \in R^{2} \mid-1<x_{1}<\right.$ $\left.+1,-\infty<x_{2}<+\infty\right\}$ which can be written

$$
A=\left\{x \in R^{2} \mid h^{1}(x)<0 \text { and } h^{2}(x)<0\right\},
$$

where

$$
h^{j}(x)= \begin{cases}-1+c^{T} x & \text { if } j=1 \\ -1-c^{T} x & \text { if } j=2,\end{cases}
$$

in which $c^{T}=\left[\begin{array}{ll}1 & 0\end{array}\right]$. Fix $\theta \geq 0$. We seek a state feedback control

$$
u \in \chi(x)
$$

(interpreted as a closed loop control $\bar{\phi}($.$) for a given initial$ state $x_{0} \in A$ ) to maximize the cost

$$
\inf _{v \in \mathcal{V}} J\left(\phi(v(.)), v(.) ; x_{0}\right)
$$

over closed loop controls $\phi(.) \in \Phi$, where $J\left(., . ; x_{0}\right)$ is the payoff, given by (3).

The number of state constraint functionals $r$ is 2. Following the proposed approach, we need to consider two associated optimal control problems $\left(P_{x_{0}}^{1}\right)$ and $\left(P_{x_{0}}^{2}\right)$ with state constraint sets

$$
\begin{aligned}
A^{1}= & \left\{x \in R^{2} \mid h^{1}(x)<0\right\}=\left\{\left(x_{1}, x_{2}\right) \mid\right. \\
& \left.-\infty<x_{1}<1 \text { and }-\infty<x_{2}<+\infty\right\}, \\
A^{2}= & \left\{x \in R^{2} \mid h^{2}(x)<0\right\}=\left\{\left(x_{1}, x_{2}\right) \mid\right. \\
& \left.-1<x_{1}<+\infty \text { and }-\infty<x_{2}<+\infty\right\}
\end{aligned}
$$

respectively. The distinguished control value for $\left(P_{x_{0}}^{1}\right)$ is $u_{1}=$ +1 (maximize the rate of outflow to prevent overflow) and that for $\left(P_{x_{0}}^{2}\right)$ is $u_{2}=-1$ (minimize the rate of outflow to prevent emptying). The two control problems are

$$
\begin{aligned}
& \left(P_{x_{0}}^{1}\right)\left\{\begin{array}{l}
\text { Minimize } \int_{0}^{\tau}\left(\frac{1}{2}|v|^{2}+\theta\right) d t \\
\text { over } \tau \geq 0 \text { and } v \in L^{2}([0, \tau] ; R) \text { satisfying } \\
\dot{x}=F x+g(-1+v) \text { a.e. } t \in[0, \tau] \\
x(0)=x_{0}, x(\tau) \in\{+1\} \times R,
\end{array}\right. \\
& \left(P_{x_{0}}^{2}\right)\left\{\begin{array}{l}
\text { Minimize } \int_{0}^{\tau}\left(\frac{1}{2}|v|^{2}+\theta\right) d t \\
\text { over } \tau \geq 0 \text { and } v \in L^{2}([0, \tau] ; R) \text { satisfying } \\
\dot{x}=F x+g(+1+v) \text { a.e. } t \in[0, \tau] \\
x(0)=x_{0}, x(\tau) \in\{-1\} \times R .
\end{array}\right.
\end{aligned}
$$

Let us now examine the value functions $V^{1}($.$) and V^{2}($. of these optimal control problems. For $j=1,2$ define the effective domain $A_{\mathrm{dom}}^{j}$ of $V^{j}($.$) to be$

$$
A_{\mathrm{dom}}^{j}:=\left\{x \in A^{j} \mid V^{j}(x)>0\right\},
$$

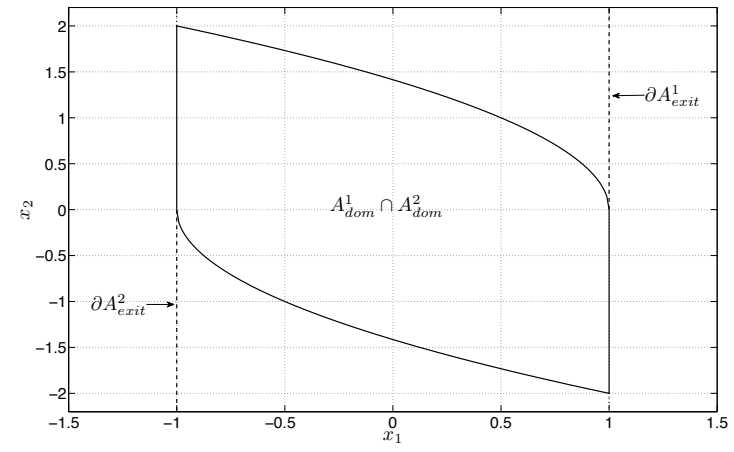

Fig. 1. The sets $\partial A_{\text {exit }}^{1}, \partial A_{\text {exit }}^{2}$ and $A_{\text {dom }}^{1} \cap A_{\text {dom }}^{2}$

Then

$$
A_{\mathrm{dom}}^{1}= \begin{cases}A^{1} & \text { if } \theta>0 \\ \left\{\left(x_{1}, x_{2}\right) \in R^{2} \mid x_{1}<1-p\left(x_{2}\right)\right\} & \text { if } \theta=0 .\end{cases}
$$

and

$A_{\mathrm{dom}}^{2}= \begin{cases}A^{2} & \text { if } \theta>0 \\ \left\{\left(x_{1}, x_{2}\right) \in R^{2} \mid x_{1}>-1-p\left(x_{2}\right)\right\} & \text { if } \theta=0 .\end{cases}$

where $p\left(x_{2}\right)=\frac{1}{2}\left(\max \left\{x_{2}, 0\right\}\right)^{2}$. Fig. 1 shows the exit boundaries of $A^{1}$ and $A^{2}$, and also, in the case $\theta=0$, the intersection $A_{\mathrm{dom}}^{1} \cap A_{\mathrm{dom}}^{2}$ of the effective domains. Notice that, if $\theta=0$, the set $A_{\mathrm{dom}}^{1}$ is a strict subset of $A^{1}$. The points in the complementary set $A^{1} \backslash A_{\text {dom }}^{1}$ are precisely those initial states such that the corresponding state trajectory exits from $A^{j}$, when we choose $u(.) \equiv+1$ and $v(.) \equiv 0$. (The surge tank overflows if the initial rate of change of fluid level is sufficiently large, even for zero 'disturbance' $v($.$) .) Similar$ remarks apply to $A_{\mathrm{dom}}^{2}$. Reference was made in Section 4 to the exit boundary $\partial A_{\text {exit }}^{j}$ of $A^{j}$, for $j=1,2$. This is the subset of the boundary of $A^{j}$ comprising points of first exit from $A^{j}$, for some state trajectory with initial state in $A^{j}$. In the present context

$\partial A_{\text {exit }}^{1}=\{+1\} \times[0,+\infty)$ and $\partial A_{\text {exit }}^{2}=\{-1\} \times(-\infty, 0]$.

(The rate of change of level cannot be strictly negative at the first time that the surge tank overflows.)

Application of the free time Maximum Principle and analysis of the extremality conditions establishes that, for $j=1,2$, and given initial state $x_{0} \in A^{j}$, there is a unique extremal. Since $\left(P_{x_{0}}^{j}\right)$ has a minimizer, the extremal is this minimizer. Semi-explicit formulae can then be obtained for the minimizing control and the value function following a change of coordinates. The change of coordinates employs the mappings $\eta^{1}:(0, \infty) \times(0, \infty) \rightarrow R^{2}$ and $\eta^{2}:(0, \infty) \times(0, \infty) \rightarrow R^{2}:$

$\eta^{1}(q, \tau)=\left(1-\frac{1}{2} \tau^{2}+\frac{1}{6} q \tau^{3}-\theta \tau / q, \tau-\frac{1}{2} q \tau^{2}+\theta / q\right)$, $\eta^{2}(r, \sigma)=\left(-1+\frac{1}{2} \sigma^{2}-\frac{1}{6} r \sigma^{3}+\theta \sigma / r,-\sigma+\frac{1}{2} r \sigma^{2}-\theta / r\right)$.

It can be shown that there exist open sets $\mathcal{O}^{j}, j=1,2$, such that, for each $j, \eta^{j}$ (.) is one-to-one on $\mathcal{O}^{j}$ and

$$
\eta^{j}\left(\mathcal{O}^{j}\right)=A_{\mathrm{dom}}^{j} .
$$


It can be shown furthermore that, for any initial state $x \in$ $A_{\mathrm{dom}}^{1}$, the value function, value function gradient, first exit time from $A_{\mathrm{dom}}^{1}$ and optimal control for problem $\left(P_{x}^{1}\right)$ are

$$
\left\{\begin{array}{l}
V^{1}(x)=\frac{1}{6} q^{2} \tau^{3}+\theta \tau \\
\nabla_{x} V^{1}(x)=(-q,-q \tau) \\
\text { first exit time from } A_{\text {dom }}^{1} \text { is }=\tau \\
v(t)=q(\tau-t), \quad \text { for a.e. } t \in[0, \tau],
\end{array}\right.
$$

in which $(q, \tau)=\left(\eta^{1}\right)^{-1}(x)$.

Also, for any initial state $x \in A_{\mathrm{dom}}^{2}$, the value function, value function gradient, first exit time from $A_{\mathrm{dom}}^{2}$ and optimal control for problem $\left(P_{x}^{2}\right)$ are

$$
\left\{\begin{array}{l}
V^{2}(x)=\frac{1}{6} r^{2} \sigma^{3}+\theta \sigma \\
\nabla_{x} V^{2}(x)=(r, r \sigma) \\
\text { fyrst exit time from } A_{\text {dom }}^{2} \text { is }=\sigma \\
v(t)=-r(\sigma-t), \quad \text { for a.e. } t \in[0, \sigma],
\end{array}\right.
$$

in which $(r, \sigma)=\left(\eta^{2}\right)^{-1}(x)$.

We show below that all the conditions are satisfied under which the solution has the structure described in Section 5. It follows that, for a given initial state, any closed loop control $\phi($.$) compatible with the following set valued feedback$ function $\bar{\chi}($.$) is maximizing:$

$$
\bar{\chi}(x)= \begin{cases}\{+1\} & \text { if } V^{1}(x)<V^{2}(x) \\ {[-1,+1]} & \text { if } V^{1}(x)=V^{2}(x) \\ \{-1\} & \text { if } V^{1}(x)>V^{2}(x) .\end{cases}
$$

for $x \in A$. The switching set $\Sigma$, for several values of the parameter $\theta$ is shown in Fig. 2. The switching set, the graph

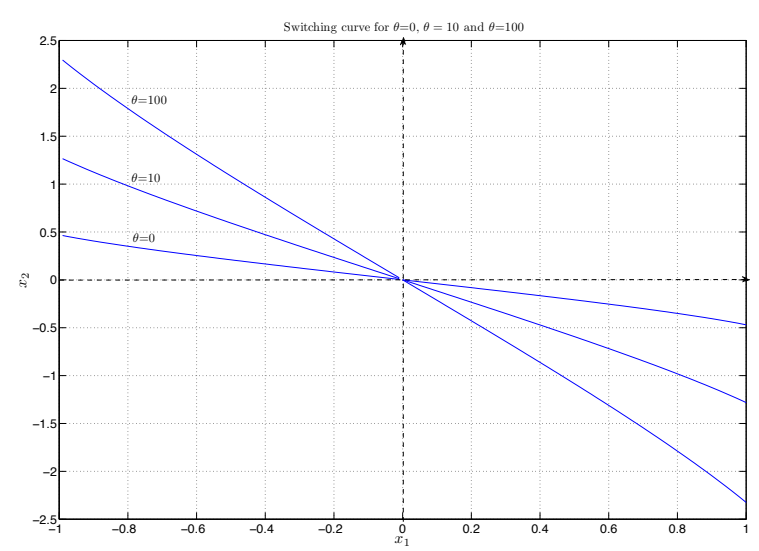

Fig. 2. The switching curve for different values of $\theta$

of a smooth curve in $A$, is

$$
\Sigma=\left\{x \in A \mid \frac{1}{6} q^{2} \tau^{3}+\theta \tau=\frac{1}{6} r^{2} \sigma^{3}+\theta \sigma\right\},
$$

in which $(q, \tau)=\left(\eta^{1}\right)^{-1}(x)$ and $(r, \sigma)=\left(\eta^{2}\right)^{-1}(x)$. In the case $\theta=0$, the switching curve is given by the following parametric formula

$$
\begin{aligned}
& x_{1}(\alpha)=\frac{\left(1+3 \alpha-2 \alpha^{2}+2 \alpha^{3}-3 \alpha^{4}-\alpha^{5}\right)}{\left(1+3 \alpha-2 \alpha^{2}-2 \alpha^{3}+3 \alpha^{4}+\alpha^{5}\right)} \\
& x_{2}(\alpha)=\frac{\sqrt{12} \alpha(\alpha-1)}{(1+\alpha)^{1 / 2}\left(1+3 \alpha-2 \alpha^{2}-2 \alpha^{3}+3 \alpha^{4}+\alpha^{5}\right)^{1 / 2}}
\end{aligned}
$$

(the denominators are positive for all non-negative $\alpha$ 's), in which $\alpha$ ranges over the interval $\frac{4}{3+\sqrt{17}}<\alpha<\frac{3+\sqrt{17}}{4}$.

Let us examine the validity of the conditions, on which the solution to the surge tank controller design problem depends. Properties $(C 2),(C 3),(C 4)$ are that the two value functions have the specified regularity, satisfy the Hamilton Jacobi equations and vanish on their exit boundaries. All of these properties can be checked from the semi-explicit formulae for the value functions and the fact that the Jacobians of the transformations $\eta^{1}($.$) and \eta^{2}($.$) are everywhere nonsingular on$ $(0, \infty) \times(0, \infty)$. Property $(C 5)$, namely that the minimizing state trajectory $x($.$) for \left(P_{x_{0}}^{1}\right)$ with initial state $x_{0} \in A$ satisfying $V^{1}\left(x_{0}\right)=V\left(x_{0}\right)$ does not cross the boundary of $A^{2}$, is illustrated by the plots in Fig. 3 for the case $\theta=0$; it follows from the facts that the switching curve passes through the origin and has negative slope and a trajectory can only leave $A_{\mathrm{dom}}^{1} \cap A_{\mathrm{dom}}^{2}$ at a point in the exit boundary of either $A^{1}$ or $A^{2}$.

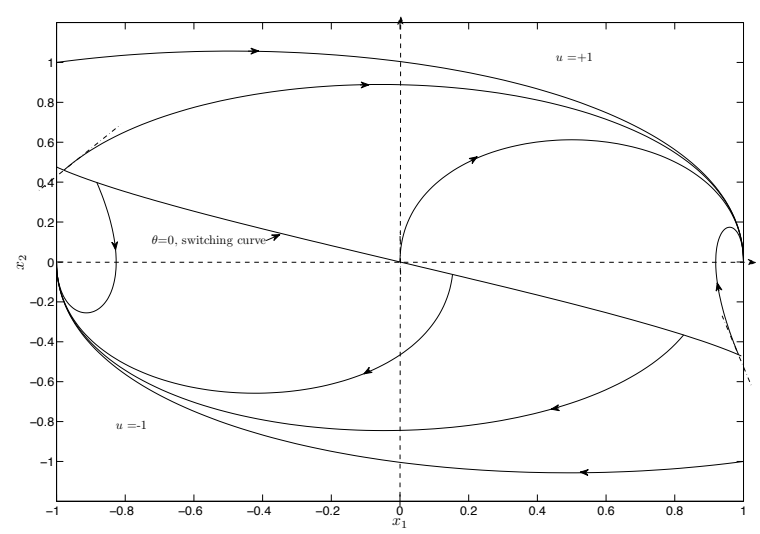

Fig. 3. Optimal trajectories

Consider now property $(C 1)$. We need to show that

$$
\left\{\begin{array}{l}
d x(t) / d t=\left[\begin{array}{ll}
0 & 1 \\
0 & 0
\end{array}\right] x(t)-\left[\begin{array}{l}
0 \\
1
\end{array}\right] u(t) . \\
y(t)=\left[\begin{array}{ll}
1 & 0
\end{array}\right] x(t)
\end{array}\right.
$$

is monotone in the following sense: fix an initial state $x_{0} \in R^{2}$. Let $u($.$) and u^{\prime}($.$) be two control functions such$ that $u(t) \leq u^{\prime}(t)$ for a.e. $t$. Write $y($.$) and y^{\prime}($.$) for the$ corresponding outputs for initial state $x_{0}$. Then $y(t) \geq y^{\prime}($.$) .$ This can be checked directly. Alternatively, it can also be deduced from sufficient conditions for monotonicity of linear systems in [16].

Finally consider property $(C 6)$. Suppose that $x$ is a point in $A$ such that $V^{1}(x)=V^{2}(x)$. The fact that the switching 
line lies inside $A_{\mathrm{dom}}^{1} \cap A_{\mathrm{dom}}^{2}$ implies $V^{1}(x)>0$. It remains to show that, if $u \in \Omega$ and $v \in R^{n}$ are points such that

$$
\nabla_{x} V^{1}(x)(f(x, u)+\sigma(x) v)=\nabla_{x} V^{2}(x)(f(x, u)+\sigma(x) v)
$$

then

$$
\nabla_{x} V^{1}(x)(f(x, u)+\sigma(x) v) \geq-\frac{1}{2}|v|^{2}-\theta .
$$

Let $(q, \tau) \in \mathcal{O}^{1}$ and $(r, \sigma) \in \mathcal{O}^{2}$ be the unique pairs of positive numbers such that $x=\eta^{1}(q, \tau)$ and $x=\eta^{2}(r, \sigma)$ respectively. Then

$$
\begin{array}{r}
\left(\nabla_{x} V^{1}(x)-\nabla_{x} V^{2}(x)\right)(f(x, u)+\sigma(x) v)= \\
=[(-q,-q \tau)-(r, r \sigma)]\left[\begin{array}{c}
x_{2} \\
v-u
\end{array}\right] .
\end{array}
$$

So, from (8), $(q+r) x_{2}+(q \tau+r \sigma)(v-u)=0$. It follows that $v-u=-\frac{(q+r)}{q \tau+r \sigma} x_{2}$.

Then

$$
\begin{aligned}
\nabla_{x} V^{1}(x)(f(x, u)+\sigma(x) v) & =[-q,-q \tau]\left[\begin{array}{c}
x_{2} \\
-\frac{(q+r) x_{2}}{q \tau+r \sigma}
\end{array}\right] \\
& =\frac{x_{2} q r(\tau-\sigma)}{q \tau+r \sigma}
\end{aligned}
$$

Claim. If $x_{2} \geq 0$ then $\tau \geq \sigma$, and if $x_{2} \leq 0$ then $\tau \leq \sigma$. If the claim is true then $\nabla_{x} V^{1}(x)(f(x, u)+\sigma(x) v) \geq 0$, from (10). This will imply (9).

We verify the claim. Since $x=\eta^{1}(q, \tau)=\eta^{2}(r, \sigma)$ we have

$$
x_{2}=\tau\left(1-\frac{1}{2} q \tau+\theta /(q \tau)\right)=-\sigma\left(1-\frac{1}{2} \sigma r+\theta /(r \sigma)\right) \text {. }
$$

Since $V^{1}(x)=V^{2}(x)$

$$
\tau\left[\frac{1}{6}(q \tau)^{2}+\theta\right]=\sigma\left[\frac{1}{6}(r \sigma)^{2}+\theta\right] .
$$

Consider the case $x_{2} \geq 0$. By (11)

$$
(q \tau)^{2}-2(q \tau)-2 \theta \leq 0 \quad \text { and } \quad(r \sigma)^{2}-2(r \sigma)-2 \theta \geq 0 .
$$

It follows that $q \tau \leq 1+(1+2 \theta)^{\frac{1}{2}} \leq r \sigma$. But then $\frac{1}{6}(q \tau)^{2}+\theta \leq$ $\frac{1}{6}(r \sigma)^{2}+\theta$. So, by (12), $\tau \geq \sigma$. The claim is confirmed in the case $x_{2} \geq 0$. The case $x_{2} \leq 0$ is dealt with similarly.

\section{Generalizations to Higher Order Problems}

The surge tank controller design problem has been formulated as a differential game in which the objective is to maximize the occupancy of a safe region $A$ in the state space. It has been solved by showing that, for this differential game, conditions $(\mathrm{C} 1)-(\mathrm{C} 6)$ are satisfied, under which the differential game decomposes into a collection of simpler, (one player) optimal control problems. The underlying methodology can be used to generate solutions to other differential games arising in controller design, when conditions (C1) - (C6) are satisfied, or to generate sub-optimal solutions and upper bounds to the value of the differential game in some circumstances when the conditions cannot be directly validated. We now discuss the broader applicability of the approach.

(C2) is a differentiability condition on the value functions of the individual optimal control problems, on their effective domains. (The requirements embodied in (C3) and (C4) that the value functions also satisfy the relevant Hamilton Jacobi equations and boundary conditions follow automatically from the hypotheses ( $\mathrm{H} 1)$ and $(\mathrm{H} 2)$.) The failure of value functions to be differentiable is typically associated with the existence of multiple minimizers and with bang-bang optimal controls. Bearing in mind that the optimal control problems considered here have dynamics linear in the control $v$ and a cost quadratic in $v$ (features that exclude bang-bang optimal control), we would expect the value functions to exhibit similar differentiability properties as those for surge tank control, in some other cases of interest. (The relevant optimal controls here are for the $v$ players in the optimal control problems, which are smooth; it is the optimal control for the $u$ player that is bang bang.) We focus attention on the remaining conditions (C1), (C5) and (C6).

Clearly condition (C1) (and the closely related condition (C5)) have key roles in the applicability of the methodology. (C1) requires that the safe region $A$ can be expressed as the intersection of $r$ sets, each involving a single state constraint functional $h^{j}($.$) , and that each of the r$ control systems associated with a particular constraint, namely

$$
\left\{\begin{array}{l}
\dot{x}(t)=f(x(t), u(t))+\sigma(x(t)) v(t) \\
x(0)=x_{0} \\
y(t)=h^{j}(x(t))
\end{array}\right.
$$

defines a monotone mapping $u(.) \rightarrow y($.$) in the sense that, for$ every

$$
\text { ' } u(t) \preceq u^{\prime}(t) \text { for all } t \text { ' implies ' } y(t) \preceq y^{\prime}(t) \text { for all } t \text { ' , }
$$

and that there is a distinguished control value $u_{j}$ such that the constant control $\bar{u}(.) \equiv u_{j}$ is maximal w.r.t. the relevant ordering $\preceq$ on the space of $u($.$) 's, used in the definition on$ monotonicity.

This monotonicity property has been identified in a number of dynamic systems of interest (besides flow systems), notably in systems biology. The literature provides general, directly verifiable, conditions for system monotonicity (of which 'positivity' is a special case). Conditions for monotonicity of linear systems are available in the monograph [16]. Criteria for monotonicity in a broader context are established by Sontag and Angeli [2], illustrated by application to the MAPK cascade class of models used to model cell signal transduction processes.

Condition (C6) excludes situations in which the disturbance signal can significantly reduce the occupancy of the safe region by forcing the state to dwell in the switching region. Validity of this condition in the surge tank control case does not appear to be anomalous. Even in situations where (C6) cannot be verified, the methodology still yields at least a sub-optimal control and an upper bound on the value of the differential game. This is because the characterization of the value function (6) is the result of combining two inequalities, proved in the Appendix. If (C6) is not confirmed, there still remains

$$
\max _{\phi \in \Phi} \inf _{v \in \mathcal{V}} J(\phi(v(.)), v(.) ; x) \leq V(x) \text { for all } x \in A
$$


Thus the pointwise infimum of the value functions for the associated optimal control problems provides the upper bound.

As an illustration of the broader application of the methodology, consider the differential game with state dimension 3 , in which the dynamics are taken to be:

$$
\dot{x}=\left[\begin{array}{ccc}
0 & 1 & 0 \\
0 & 0 & 1 \\
0 & 0 & -1
\end{array}\right] x-\left[\begin{array}{l}
0 \\
1 \\
0
\end{array}\right] u+\left[\begin{array}{l}
0 \\
0 \\
1
\end{array}\right] v,
$$

the $u$ control is constrained to lie in the set $\Omega=[-1,+1]$ and the state constraint set is

$$
A=\left\{x \in R^{3} \mid-1 \leq x_{1} \leq+1\right\} .
$$

As we have noted, the differential game for the surge tank control problem is associated with the small noise stochastic model

$$
d x(t)=\left[\begin{array}{ll}
0 & 1 \\
0 & 0
\end{array}\right] x(t) d t+\left[\begin{array}{l}
0 \\
1
\end{array}\right]\left(-u(t) d t+\epsilon^{\frac{1}{2}} d B(t)\right) .
$$

The modified differential game corresponds to replacing the white noise disturbance $d B(t)$ by colored noise $e(t)$, generated by the stochastic differential equation

$$
d e(t)=-e d t+\epsilon^{\frac{1}{2}} d B(t) .
$$

There are two optimal control problems to consider, associated with the state constraint sets

$$
\begin{aligned}
& A^{1}=\left\{x \in R^{3} \mid-\infty<x_{1}<+1\right\} \text { and } \\
& A^{2}=\left\{x \in R^{3} \mid-1<x_{1}<+\infty\right\}
\end{aligned}
$$

with boundaries

$$
\partial A^{1}=\{+1\} \times R \times R \text { and } \partial A^{2}=\{-1\} \times R \times R .
$$

The controls that drive the state away from the boundaries of $A^{1}$ and $A^{2}$ are $u_{1}=+1$ and $u_{2}=-1$ respectively.

The monotonicity condition (C1) is satisfied. This may be verified directly, or by application of the sufficient condition ([16], Corollary III.3). It is not possible to confirm condition (C6) analytically. But, even if it is violated, our decomposition technique yields sub-optimal control strategies and bounds on the upper value of the differential game, as described above. A numerical implementation of the technique is as follows:

Let $h$ be the sample period. At time $t_{k}=k h$ measure the state $x\left(t_{k}\right)$ and compute the $r$ (optimal control problem) minimum costs:

$$
\begin{aligned}
V_{\text {min }}^{j}\left(x\left(t_{k}\right)\right)= & \operatorname{Min} \frac{1}{2} \int_{0}^{\tau}\left(|v|^{2}+\theta\right) d t \\
& \dot{x}=f\left(x, u_{j}\right)+\sigma(x) v, \\
& x(0)=x\left(t_{k}\right), x(\tau) \in \partial A^{j}
\end{aligned}
$$

Choose

$$
u(t)=u_{j} \text { on }[k h,(k+1) h]
$$

where

$$
j=\arg \min _{j^{\prime}}\left\{V_{\min }^{j^{\prime}}\left(x\left(t_{k}\right)\right)\right\} .
$$

The two optimization problems involved are solved for initial states on a grid in the 'box' $\left\{\left(x_{1}, x_{2}, x_{3}\right) \mid-1 \leq x_{1} \leq+1,-L \leq x_{2} \leq+L,-L \leq x_{3} \leq\right.$ $+L\}$ for some suitably large bound $L$. The minimum costs are compared and the feedback control value is chosen to be +1 or -1 , depending on which is the larger. The switching surface is illustrated in Figure 4.

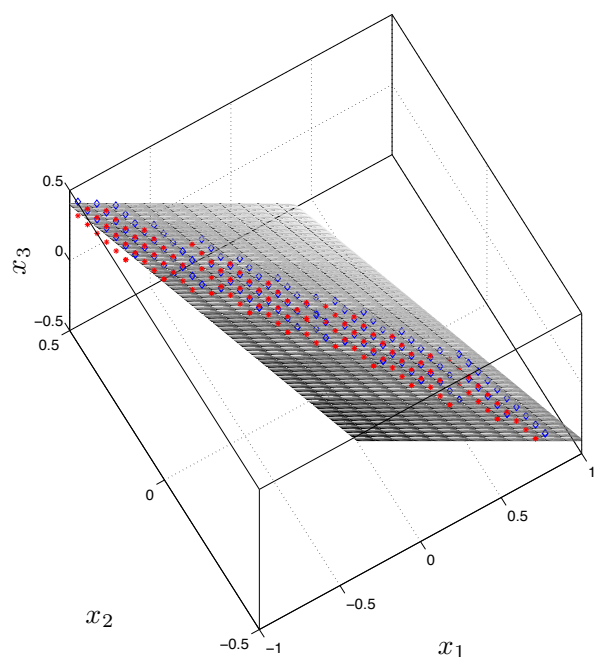

Fig. 4. The switching surface in $3 \mathrm{D}$ space

\section{APPENDIX A}

\section{PROOF OF THEOREM 5.1}

Attention is limited to the case $r=2$ (which in particular covers the surge tank controller design problem). The general situation $r \geq 2$ is dealt with by means of the $r=2$ analysis coupled with an induction on $r$. Fix an initial state $x_{0} \in A$. We shall show:

(A): For any $\phi(.) \in \Phi$,

$$
\inf _{v \in \mathcal{V}} J\left(\phi(v(.)), v(.) ; x_{0}\right) \leq V\left(x_{0}\right)
$$

and

(B): For any closed loop control $\bar{\phi}(.) \in \Phi$ compatible with (7) and any $v \in \mathcal{V}$,

$$
J\left(\bar{\phi}(v(.)), v(.) ; x_{0}\right) \geq V\left(x_{0}\right) .
$$

Indeed, the first relation implies that $V\left(x_{0}\right) \quad \geq$ $\inf _{v \in \mathcal{V}} J\left(\bar{\phi}(v()),. v(.) ; x_{0}\right)$, while the second relation implies that $\inf _{v \in \mathcal{V}} J\left(\bar{\phi}(v()),. v(.) ; x_{0}\right) \geq V\left(x_{0}\right)$. We conclude from these last two relations that

$$
\inf _{v \in \mathcal{V}} J\left(\bar{\phi}(v), v ; x_{0}\right)=\max _{\phi(.) \in \Phi} \inf _{v \in \mathcal{V}} J\left(\phi(v), v ; x_{0}\right)=V\left(x_{0}\right) .
$$

Since the initial state $x_{0}$ is arbitrary, this tells us that $V($.$) is$ the upper value of the differential game, and any closed loop control $\bar{\phi}($.) compatible with (7) (for the given initial state) is optimal.

Consider first (A). Select any $\phi(.) \in \Phi$ and take any $j \in$ $\arg \min _{j^{\prime}}\left\{V^{j^{\prime}}\left(x_{0}\right)\right\}$. The optimal control problem $\left(P_{x_{0}}^{j}\right)$ has a minimizer $\bar{v}($.$) , with state trajectory \bar{x}($.$) and first exit time$ $\bar{\tau}$ from $A^{j}$. (See the comments at the end of Section 3.) By $(C 5), \bar{\tau}$ is the first exit time also from $A$. Let $u=\phi(\bar{v})$ and write $x($.$) for the state trajectory obtained by solving \dot{x}(t)=$ $f(x(t), u(t))+\sigma(x) \bar{v}(t)$ with initial value $x_{0}$. From $(C 1), x($. 
has a first exit time $\tau$ from $A$ and $\tau \leq \bar{\tau}$. But then

$$
\begin{aligned}
\inf _{v \in \mathcal{V}} J\left(\phi(v), v ; x_{0}\right) & \leq J\left(u, \bar{v} ; x_{0}\right)=\frac{1}{2} \int_{0}^{\tau}|\bar{v}|^{2} d t+\theta \tau \\
& \leq \frac{1}{2} \int_{0}^{\bar{\tau}}|\bar{v}|^{2} d t+\theta \bar{\tau} \\
& =V^{j}\left(x_{0}\right)=V\left(x_{0}\right) .
\end{aligned}
$$

Relation (14) is confirmed.

Consider next (B). Let $\bar{\phi}($.$) be any closed loop control$ compatible with (7). Take any $v \in \mathcal{V}$. Write $u()=.\bar{\phi}(v()$. and $x($.$) for the solution to \dot{x}(t)=f(x(t), u(t))+\sigma(x(t)) v(t)$ with initial state $x_{0}$. We may assume that $x($.$) has a first exit$ time $\tau_{e}$ from $A$ since, otherwise, (15) is automatically satisfied. We may also assume that $V\left(x_{0}\right)>0$. If $V(x(t))>0$ for all $t<\tau_{e}$, write $\tau=\tau_{e}$. Otherwise we may define $\tau$ to be the infimum of times $\tau^{\prime} \geq 0$ such that $V(x(t))>0$ for $t<\tau^{\prime}$. Since $V$ is continuous, and in view of $(C 3)$ we have, in either case, that $\tau>0$,

$$
\lim _{t \uparrow \tau} V(x(t))=0
$$

and

$$
V(x(t))>0 \text { for all } t \in(0, \tau) .
$$

The function $V($.$) is Lipschitz continuous on compact subsets$ of $A_{\mathrm{dom}}^{1} \cap A_{\mathrm{dom}}^{2}$, as a lower envelope of continuously differentiable functions on the open set $A_{\mathrm{dom}}^{1} \cap A_{\mathrm{dom}}^{2}$. Take any sequence of positive numbers $\tau_{i} \uparrow \tau$. It follows that $t \rightarrow V(x(t))$ is an absolutely continuous function on $\left[0, \tau_{i}\right]$. In view of (16), we may write

$$
V\left(x_{0}\right)=0-\lim _{i \rightarrow \infty} \int_{0}^{\tau_{i}} d / d t V(x(t)) d t .
$$

Take $\mathcal{T}$ to be the set of times $t \in(0, \tau)$ such that

(i): $t$ is a point of differentiability of $x(),. t^{\prime} \rightarrow V\left(x\left(t^{\prime}\right)\right)$ and of $t^{\prime} \rightarrow V^{j}\left(x\left(t^{\prime}\right)\right)$ for $j=1,2$.

(ii): $\dot{x}(t)=f(x(t), u(t))+\sigma(x(t)) v(t)$ and $u(t) \in \Omega$.

The set $\mathcal{T}$ so defined has full measure. For any $t \in \mathcal{T}$ we have

$$
d / d t V(x(t))=\lim _{i \rightarrow \infty} \epsilon_{i}^{-1}\left[V\left(x\left(t+\epsilon_{i}\right)\right)-V(x(t))\right] .
$$

for some $\epsilon_{i} \downarrow 0$. Since the $V^{j}$ 's are continuous, we can arrange, by extracting a subsequence, that, for some $j$, $V^{j}(x(t))=V(x(t))$ and

$$
d / d t V(x(t))=\lim _{i \rightarrow \infty} \epsilon_{i}^{-1}\left[V^{j}\left(x\left(t+\epsilon_{i}\right)\right)-V^{j}(x(t))\right] .
$$

Since $V^{j}($.$) is differentiable at x(t)$ (see $(C 2)$ ), and $x($.$) is$ differentiable at $t$ with derivative $f(x(t), u(t))+\sigma(x(t)) v(t)$, we have

$$
d / d t V(x(t))=\nabla_{x} V^{j}(x(t))(f(x(t), u(t))+\sigma(x(t)) v(t)) .
$$

Now consider the partition of $\mathcal{T}$ into three disjoint sets $\mathcal{T}=$ $\mathcal{T}_{1} \cup \mathcal{T}_{2} \cup \mathcal{T}_{3}$ :

$$
\begin{aligned}
\mathcal{T}_{1}= & \left\{t \in \mathcal{T} \mid V^{1}(x(t)) \neq V^{2}(x(t))\right\} \\
\mathcal{T}_{2}= & \left\{t \in \mathcal{T} \mid V^{1}(x(t))=V^{2}(x(t))\right. \text { and } \\
& \left(\nabla_{x} V^{1}(x(t))-\nabla_{x} V^{2}(x(t))\right)(f(x(t), u(t)) \\
& +\sigma(x(t)) v(t))=0\} \\
\mathcal{T}_{3}= & \left\{t \in \mathcal{T} \mid V^{1}(x(t))=V^{2}(x(t))\right. \text { and } \\
& \left(\nabla_{x} V^{1}(x(t))-\nabla_{x} V^{2}(x(t))\right)(f(x(t), u(t)) \\
& +\sigma(x(t)) v(t)) \neq 0\} .
\end{aligned}
$$

Take any $t \in \mathcal{T}_{3}$. We deduce from the defining properties (i) and (ii) for $\mathcal{T}_{3}$ that

$$
V^{1}(x(s)) \neq V^{2}(x(s)) \text { for all } s \in(t-\delta, t+\delta) \backslash\{t\},
$$

for some $\delta>0$. But this means that $\mathcal{T}_{3}$ is a set of isolated points; that is, each $t \in \mathcal{T}_{3}$ is contained in an open set disjoint from $\mathcal{T}_{3} \backslash\{t\}$. Such sets are countable and therefore have zero measure. We may therefore redefine the full-measure set $\mathcal{T}$ to exclude $\mathcal{T}_{3}$. Now $\mathcal{T}=\mathcal{T}_{1} \cup \mathcal{T}_{2}$.

Take any $t \in \mathcal{T}$. We claim that

$$
d / d t V(x(t)) \geq-1 / 2|v(t)|^{2}-\theta .
$$

There are two cases to consider:

1. $t \in \mathcal{T}_{1}$. We may assume without loss of generality that $V^{1}(x(t))<V^{2}(x(t))$. Then $V^{1}(x(t))=V(x(t))$ and $u(t)=$ $u_{1}$, since $\bar{\phi}($.$) is compatible with (7). It follows from (C 2)$ and $(C 5)$ that

$$
\begin{aligned}
d / d t V(x(t)) & =\nabla_{x} V^{1}(x(t))\left(f\left(x(t), u_{1}\right)+\sigma(x(t)) v(t)\right) \\
& \geq-1 / 2|v(t)|^{2}-\theta
\end{aligned}
$$

as required.

2. $t \in \mathcal{T}_{2}$. Now $V^{1}(x(t))=V^{2}(x(t))$. We know from the second defining property of $\mathcal{T}_{2}$ that

$$
\begin{aligned}
d / d t V(x(t)) & =\nabla_{x} V^{1}(x(t))(f(x(t), u(t))+\sigma(x(t)) v(t)) \\
& =\nabla_{x} V^{2}(x(t))(f(x(t), u(t))+\sigma(x(t)) v(t)) .
\end{aligned}
$$

But then, by (C6),

$$
\begin{aligned}
d / d t V(x(t)) & =\nabla_{x} V^{1}(x(t))(f(x(t), u(t))+\sigma(x(t)) v(t)) \\
& \geq-\frac{1}{2}|v(t)|^{2}-\theta .
\end{aligned}
$$

This validates the claim. Finally, we deduce from (18) that

$$
\begin{aligned}
V\left(x_{0}\right) & =-\lim _{i \rightarrow \infty} \int_{0}^{\tau_{i}} d / d t V(x(t)) d t \\
& \leq \frac{1}{2} \int_{0}^{\tau}|v(t)|^{2} d t+\theta \tau=J\left(u=\bar{\phi}(v), v ; x_{0}\right) .
\end{aligned}
$$

Relation (15) is confirmed.

\section{ACKNOWLEDGEMENT}

This research was supported in part by EPSRC grant EP/G066477/1 and in part by Marie-Curie ITN SADCO.

\section{REFERENCES}

[1] O. AlvAREZ and M. BARDI, Viscosity Solutions Methods for Singular Perturbations in Deterministic and Stochastic Control, SIAM J. Control and Optim., 40, 2001, pp. 1159-1188.

[2] D. ANGELI and E. D. SONTAG, Monotone Control Systems, IEEE Trans. Aut. Control, 48, 2003, pp. 1684-1698.

[3] K. J. ASTROM and T. HAGglund, PID Controllers: Theory, Design, and Tuning, ISA, Research Triangle Park, North Carolina, 1995.

[4] M. BARDI and I. CAPUZZO-DOLCETTA, Optimal Control and Viscosity Solutions of Hamilton-Jacobi-Bellman Equations, Birkhäuser, Boston, 1997.

[5] P. J. CAMPO and M. MORARI, Model Predictive Optimal Averaging Level Control, AIChE Journal, 35, 1989, pp. 579-591.

[6] P. CARDALIAGUET and S. PLASKACZ, Invariant Solutions of Differential Games and Hamilton-Jacobi-Isaacs Equations for Time-Measurable Hamiltonians, SIAM J. Control Optim. 38, 2000, pp. 1501-1520.

[7] E. CARLINI, M. FALCONE and R. FERRETTI, An Efficient Algorithm for Hamilton-Jacobi Equations in High Dimension, Computing and Visualization in Science, 7, 2004, pp. 15-29

[8] T. F. CHEUNG and W. L. LUYBEN, Liquid-Level Control in Single Tanks and Cascades of Tanks with Proportional-Only and ProportionalIntegral Feedback Controllers, Industrial and Engineering Chemistry Fundamentals, 18, 1979, pp.15-21. 
[9] J. M. C. CLARK, M. R. JAMES and R. B. VINTER, The Interpretation of Discontinuous state Feedback Control Laws as Non-Anticipative Control Strategies in Differential Games, IEEE TAC, 49, 2004, pp. 1360-1365.

[10] J. M. C. Clark and R. B. VinTER, A Differential Dynamic Games Approach to Flow Control, Proc. 43rd Conference on Decision and Control, Hawaii, 2003, pp. 1228-1231.

[11] E. CRISTIANI and M. FALCONE, Numerical Solution of the Isaacs Equation for Differential Games with State Constraints , Proc. 17th IFAC World Congress, Seoul Korea, 2008.

[12] P. DUPUIS and W. M. MCENEANEY, Risk-Sensitive and Robust Escape Criteria, SIAM J. Control Optim. 35, 1997, pp. 2021-2049.

[13] M. BOUE and P. DUPUIS, Risk-Sensitive and Robust Escape Control for Degenerate Diffusion Processes, Math. of Control, Signals and Systems, 14,2001 , pp. $62-85$

[14] R. J. ELLIOTT and N. J. KALTON, The Existence of Value in Differential Games, Mem. Amer. Math. Soc., AMS, Providence, RI, 1972.

[15] M. FALCONE, A numerical approach to the infinite horizon problem of deterministic control theory, Applied Mathematics and Optimization, Springer New York, 15, 1987, pp. 1-13.

[16] L. FARINA and S. RINALDI, Positive Linear Systems: Theory and Applications, Wiley, New, York, 2000.

[17] W. H. Fleming and H. M. SONER, Controlled Markov Processes and Viscosity Solutions, Springer Verlag, New York, 1993.

[18] J. C. KANTOR, Non-linear sliding mode controller and objective function for surge tanks, Int. J. Control, 50, 1989, pp. 2025-2047.

[19] J. B. RAWLINGS and D. Q. MAYNE, Model Predictive Control: Theory and Design, Nob Hill Publishing, Madison, 2009.

[20] K. A. McDONALD, T. J. McAVOY and A. TITS, Optimal averaging level control, AIChE Journal, 32, 1986, pp. 75-86.

[21] W. M. McENEANEY, Max-Plus Methods for Nonlinear Control and Estimation, Birkhäuser, Boston, 2006.

[22] I. M. MitChell, A. M. BAYEN and C. J. TOMLIN, A Time-Dependent Hamilton-Jacobi Formulation of Reachable Sets for Continuous Dynamic Games, IEEE Trans. Aut. Control, 50, 2005, pp. 947-957.

[23] R. B. VINTER, Optimal Control, Birkhäuser, Boston, 2000.

[24] P. WhitTLE, Risk-Sensitive Optimal Control, Wiley, New York, 1990.

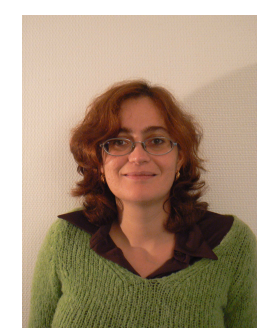

P. Falugi Paola Falugi is currently a Research Associate at Department of Electrical and Electronic Engineering, Imperial College London,UK. She received the Ph.D. degree in Systems Engineering from Università di Bologna in 2002. She spent four years with Università di Firenze, Italy, and one year with SUPELEC, France, as postdoc researcher. Her current research interests are in predictive control and control of constrained nonlinear systems, robust control and system identification.

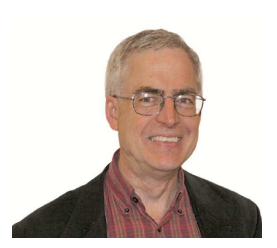

R. B. Vinter R. B. Vinter obtained a BSc in engineering science at Oxford University, a PhD in control engineering at Cambridge University and a $\mathrm{ScD}$ in mathematics from Cambridge University.

As a recipient of a Harkness Fellowship, he was a postdoctoral researcher at the Electronic Systems Laboratory, Massachusetts Institute of Technology for two years. Since 1974 he has been on the academic staff at Imperial College London, where he is now Professor Control Theory. He is Dean of the Faculty of Engineering and formerly Head of the Control and Power Group in the Department of Electrical and Electronic Engineering. His research has centered on nonlinear control, optimization, differential games, tracking and estimation.

$\mathrm{He}$ has published over 150 scientific papers and two text books.

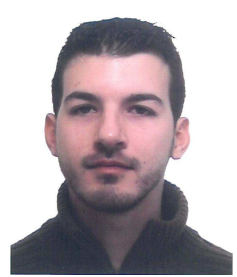

P.A. Kountouriotis obtained an M.Eng. degree (First Class) in electrical engineering and the Ph.D. degree from Imperial College London in 2005 and 2009 , respectively.

$\mathrm{He}$ is currently employed as a Research Associate in the Control and Power Group, Department of Electrical and Electronic Engineering at Imperial College. His research interests focus on theoretical and computational aspects of stochastic systems and control. 